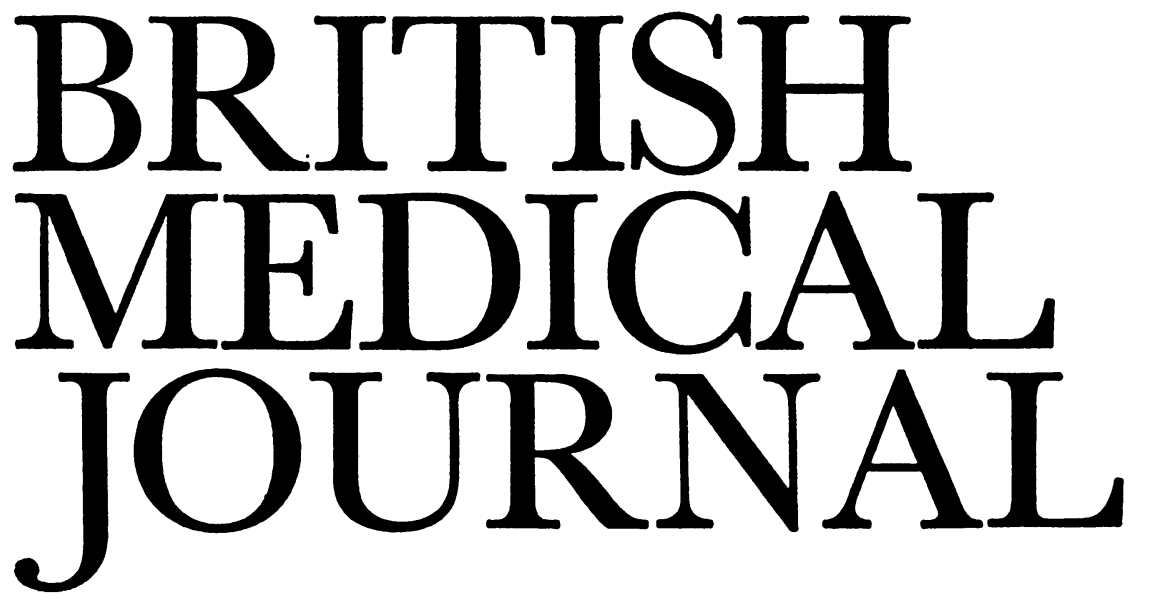

LONDON SATURDAY 8 MAY 1971

\title{
General Medical Council
}

Since 1948 polls for those elections for the General Medical Council which were contested have been around $40 \%{ }^{1-3}$ so at first sight the $25 \%$ poll reported on page 349 hardly suggests a lively interest by doctors in the recent election. ${ }^{4}$ The profession has been subjected to a steady stream of comment about every aspect of the G.M.C. from all quarters during the past three years, so there should be no reason for doctors to have been ignorant of the issues at stake. But probably the low poll was more a consequence of doctors deliberately abstaining because they knew too little about the 38 candidates to feel able to make a thoughtful choice. Certainly some of the candidates who were sponsored by other bodies ${ }^{5}$ did quite well, and this should provide food for thought at the Annual Representative Meeting at Leicester when it discusses the B.M.A. Council's proposals to rescind the current B.M.A. policy of non-involvement in G.M.C. elections (Supplement, 1 May, p. 28).

As well as this proposal the Council is also recommending the Representative Body to accept the Brynmor Jones report on the G.M.C.'s constitution, ${ }^{78}$ and to start negotiations-including a review of the G.M.C.'s functions-immediately with the Government and the G.M.C. The Council's other recommendation, that the B.M.A. should no longer oppose an annual retention fee, is a natural consequence of its acceptance of the proposed constitutional reforms. If approved, it would resolve the issue which sparked the initial disagreement between doctors and their statutory professional body. Even if agreement is reached between the profession, the Government, and the G.M.C., parliamentary time will have to be found in a heavy legislative programme for the necessary amendments to the Medical Act. Furthermore, increasing public interest in the registration of doctors and the machinery for dealing with patients' complaints could mean that amending legislation, even with Government support, might not have a quick or easy ride through Parliament.

So though there will be urgency in discussing the reform of the G.M.C. there is less need for haste in deciding exactly how the B.M.A. should participate in future elections. The Association might be wise to wait and see what will be the ultimate shape and duties of the G.M.C. before it makes any final decision about the sponsorship of candidates. If the number of elected members is to be substantially increased, with a corresponding increase in their influence on the G.M.C.'s affairs-which was the profession's objective-then the responsibility resting on an organization which supports candidates will be even greater than it has been in the past. With its object of improved elected representation in sight, the profession must think carefully about having a viable pre-election procedure which would ensure enough suitable candidates as well as an informed electorate. The results of any hastily conceived proposals-whatever medical body initiates them-might be an ineffectual G.M.C. commanding the respect of neither the public nor the profession.

1 Minutes of the General Medical Council-Vol. LXXXVIII, 1951, London 1953.

Minutes of the General Medical Council-Vol.XCIII, 1956, London, 1958. 3 Minutes of the General Medical Council-Vol. CIII, 1966, London, 1967. 4 British Medical Fournal, 1971, 1, 359.

5 On Call, March, 1971, p. 1.

- Medical World, March/April, 1971, p. 2.

- British Medical fournal Supplement, 1971, 1, 55.

8 British Medical fournal, 1971, 1, 565.

\section{Clioquinol and Neurological Disease}

A remarkable neurological syndrome described under the portmanteau term of subacute myelo-optico-neuropathy with abdominal symptoms was first recognized in Japan in the 1950s. Since then it has increased greatly in frequency. It is apparently largely restricted to Japan, where there may be at least 10,000 cases.

I. Sobue and colleagues ${ }^{1}$ have recently reviewed 752 personal cases. Most of them had a history of chronic abdominal symptoms, usually consisting of pain and diarrhoea preceding the neurological illness. There was usually a prodromal exacerbation of the abdominal symptoms before the acute or subacute onset of a sensory neuropathy in the lower limbs. In about two-thirds of the patients there were painful dysaesthesiae, and they developed an ataxic gait. Half of them developed muscular weakness, particularly of the lower limbs; a quarter had visual impairment. The follow-up of 684 of these patients extending from periods of six months to ten years showed that $19 \%$ had chronic relapses, but that at fol- 
low-up $7 \%$ were cured, $54 \%$ improved, and $25 \%$ were unchanged. The remainder were worse or had died. However, $93 \%$ of them had persisting painful dysaesthesiae. At the time of development of the neurological picture there were associated systemic disturbances with raised blood pressure and white cell count in the peripheral blood, a raised erythrocyte sedimentation rate, and glycosuria. The aetiology of this condition is not known for certain, though the persistent painful dysaesthesiae have some resemblance to those seen after thalidomide intoxication. ${ }^{2}$

Epidemiological evidence has recently been advanced by T. Tsubaki and colleagues ${ }^{3}$ associating the syndrome with clioquinol therapy. This drug is generally available in the United Kingdom and many other parts of the world as EnteroVioform and is taken by travellers to prevent minor gastrointestinal upsets. It is for this reason that attention should be directed to a syndrome which until now has been rarely recognized outside Japan. Tsubaki and colleagues studied the records of 263 patients with various digestive disorders who received clioquinol therapy, comparing them with the records of 706 patients with similar disease who did not receive clioquinol. No neurological symptoms appeared in the latter cases, but $44(16.7 \%)$ cases of subacute myelo-opticoneuropathy appeared in the clioquinol group. Of those patients receiving therapy for more than 14 days 35\% developed the syndrome, while only $2.6 \%$ of those treated for 13 days or less were afflicted. They also found that 166 of 171 patients with the neurological syndrome whom they reviewed had received clioquinol therapy for the prodromal abdominal symptoms. On the basis of these findings they implicated clioquinol as the possible cause of the neurological disease, and the Japanese Ministry of Health and Welfare withdrew permission for the production and sale of clioquinol in Japan from September 1970.

Clioquinol had previously been thought to be remarkably safe for long-term administration. For instance, in the United States L. M. Gholz and W. L. Arons ${ }^{4}$ prescribed this drug in a dose of $750 \mathrm{mg}$ daily for four years to 4,000 institutionalized patients. They reported uniform success in preventing gastrointestinal disorders and extremely rare toxic sideeffects. They did, however, note that 20 patients developed "an unusual gait change". Eighteen of them recovered completely and 13 were later able to take the drug for long periods without recurrence of the disturbance of gait. The dosage given in the Japanese cases (0.6-1.6 g per day) was of the same order as used in this American study. Nevertheless over the years since the halogenated hydroxyquinolines were introduced a number of cases of optic atrophy and occasional damage to the spinal cord have been reported. ${ }^{5-7}$ Moreover Entero-Vioform itself was said to be cause of optic atrophy in two recently reported cases from Germany. ${ }^{8}$

W. T. Cooke and W. T. Smith ${ }^{9}$ reported degeneration of the spinal cord and peripheral nerves in patients with adult coeliac disease, though the cause of the degeneration was not discovered. It is therefore possible that the relationship to clioquinol therapy in the Japanese cases may be fortuitous. For instance, Tsubaki and colleagues' control group, who received no clioquinol therapy, may have differed either in type or in severity of abdominal disorders from those receiving the drug. Nevertheless the evidence these authors advance deserves careful attention. It is possible that the abdominal condition itself which developed in these Japanese patients was responsible for abnormal sensitivity to clioquinol, or that there is an underlying genetic predisposition to intoxication with this drug in the Japanese.
More reports may soon appear linking neurological diseases with clioquinol therapy. It is important that their significance should be assessed in relation to the very large quantity of clioquinol being taken throughout the world, and that attention should be given to the underlying abdominal condition for which the drug is given and to ethnic factors.

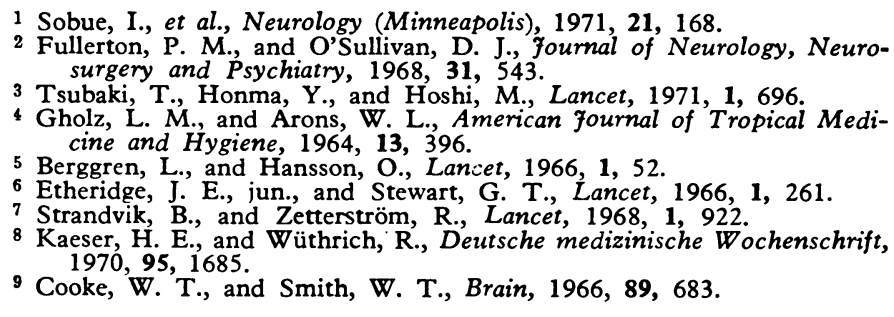

\section{Progress in Myeloma}

In recent years there has been considerable progress in our understanding of myeloma and in its treatment. Many aspects of the subject were discussed by leading authorities from all over the world at a "myeloma workshop" held recently in London, and shortened versions of most of their contributions are printed in this week's B.M.F. (page 319). All those attending the meeting agreed that it was outstanding of its kind, and its two scientific secretaries, Dr. D. A. G. Galton and Professor J. R. Hobbs, and the Leukaemia Research Fund, which sponsored it, are to be congratulated.

Myelomatosis is one of the few malignant diseases in which the cells regularly produce measurable amounts of metabolites that give some indication of the amount of tumour present. These metabolites are serum paraproteins and may be $\operatorname{IgA}, \operatorname{IgG}$, or $\operatorname{IgM}$ in type, and Hobbs has recently reviewed their significance in the disease. ${ }^{1}$ The studies reported at the workshop by J. L. Fahey and S. Salmon, together with previous ones by Hobbs and others, on the turnover rates of serum immunoglobulins indicate that most IgG and $\operatorname{IgA}$ myelomas may double their size in about 6 to 12 months and that as few as four doublings may occur from the time of early disease until death. If the growth of the tumour was constant and exponential, it could be calculated that the tumour must have started to grow some 15 to 20 years previously. Nevertheless, Salmon's measurements have shown that myeloma growth is not constant and that the rate of doubling becomes less as the number of tumour cells increases-as is the case with several other tumours in animals and man. His findings suggest that the early stages of tumour growth usually occur about five years before the disease is diagnosed.

Another topic discussed at the workshop was extramedullary plasmacytoma. In a review of 266 patients E. Wiltshaw showed that three-quarters of them had presented with a tumour of the upper respiratory tract and that the spread of the disease was much more like that of reticulum cell sarcoma than myelomatosis. The early invasion of lymph nodes was characteristic, and even when the disease became generalized the bone marrow was rarely involved. On the other hand, she emphasized that solitary myeloma of bone is merely an unusual form of myelomatosis and when disseminated is indistinguishable from the typical disease.

Another interesting discovery about myelomatosis is that in both experimental animals and in man the immunoglobulin produced by the malignant cells may have a specific affinity for a particular antigen. Thus the serum of one patient de- 